\section{Qualitative C-reactive protein as a marker of neonatal sepsis in a tertiary neonatal unit in Sudan}

\author{
Abdelmoneim E. Kheir, ${ }^{1}$ \\ Ghada A. Jobara, ${ }^{2}$ Kamal M. Elhag, ${ }^{3}$ \\ Mohamed Z. Karar ${ }^{2}$ \\ 'Department of Pediatrics, University \\ of Khartoum, Khartoum; ${ }^{2}$ Department \\ of Neonatology, Soba University \\ Hospital, Khartoum; ${ }^{3}$ Microbiology \\ Department, Soba University Hospital, \\ Khartoum, Sudan
}

\section{Abstract}

Sepsis is one of the most common causes of morbidity and mortality in newborns. Diagnosis of neonatal sepsis may be difficult because clinical presentations are often non-specific. The aim of this study was to evaluate the role of qualitative C-reactive protein in the diagnosis of neonatal sepsis, and examine the correlation between C-reactive protein, blood culture and risk factors for sepsis. This was a prospective study, conducted in the Neonatal Intensive Care Unit at Soba University Hospital, Sudan. A total of seventy babies with a clinical diagnosis of sepsis were included. Chi square test was used to determine the association between C-reactive protein and risk factors for sepsis and also the association between $\mathrm{C}$-reactive protein and blood culture. Blood culture was positive in $41.4 \%$ of babies, and C-reactive protein was positive in $58 \%$ of babies with positive blood culture. There was significant association between C-reactive protein results and blood culture $(\mathrm{P}=0.00)$. In conclusion, we can assume that $\mathrm{C}$ reactive protein is a reliable diagnostic marker of neonatal sepsis, especially in developing communities with poor resources.

\section{Introduction}

Neonatal sepsis is a clinical syndrome of bacteraemia characterized by systemic signs and symptoms of infection in the first month of life. It encompasses systemic infection of newborn including septicemia, meningitis, pneumonia, arthritis, osteomyelitis and urinary tract infection. ${ }^{1}$

The diagnosis of infection in neonates is difficult, because of the non-specific clinical presentation and the lack of reliable diagnostic tests. As a result of this uncertainty, antimicrobial chemotherapy is often commenced on the slightest clinical suspicion of infection.
Recently there has been great interest in the potential diagnostic value of a range of hematological and immunological surrogate markers of infection. ${ }^{2,3}$

Although a positive blood culture remains the standard for diagnosing neonatal sepsis, many investigators have assessed measuring the host response as an adjunct to culturebased diagnosis. The goal of serum biomarker research is to identify a means by which an infected child can be identified rapidly, before the onset of life-threatening symptoms. ${ }^{4}$

C-reactive protein (CRP), the most commonly used biomarker, is synthesized within 6 $\mathrm{h}$ of exposure to an infectious process and usually becomes abnormal within $24 \mathrm{~h}$. Because CRP takes up to $24 \mathrm{~h}$ after the onset of an infection to become abnormal, it has little utility in assisting the early detection of sepsis. Creactive protein is also limited in that other processes in addition to infection can result in elevation, including trauma and ischemia. ${ }^{5}$

The most rapid quantitative method for determining CRP concentration is by nephelometry. Alternative positive CRP latex agglutination test of undiluted sample corresponds to plasma CRP concentration of $0.6-1 \mathrm{mg} / \mathrm{dL}$. Normalization of CRP elevation appears to be helpful in determining the response to antimicrobial therapy and duration of treatment. ${ }^{6}$

Neonatal septicemia continues to be a major cause of morbidity and mortality in Sudan. It is one of the major causes of neonatal mortality in developing countries contributing to $26 \%$ of all neonatal deaths. ${ }^{7}$

In developing countries there is need for a test that is cheap and easily performed with quick availability of results. An ideal diagnostic test for neonatal sepsis should have maximum sensitivity and specificity. In recent years, various investigators have evaluated some highly sensitive and specific inflammatory markers (e.g. ELISA methods, haptoglobins, interleukins and procalcitonin) to diagnose neonatal sepsis. ${ }^{8}$ Although, these markers are sensitive and specific, they are expensive, thus not practical for developing countries. For this reason, there have been many attempts to develop screening tests to identify infected neonates and guide the duration of treatment.

The aim of this study is to i) evaluate the role of qualitative CRP in the diagnosis of neonatal sepsis, ii) determine the relationship between CRP and risk factors for sepsis and iii) examine the correlation between CRP and blood culture. To our knowledge this is the first study done in Sudan to examine the correlation between CRP and blood culture.

\section{Materials and Methods}

This was a prospective hospital-based, case
Correspondence: Abdelmoneim Elamin Kheir, Department of Pediatrics, University of Khartoum, Almc Namr street, 11115 Khartoum, Sudan.

Tel. +249.912313110 - Fax: +249.183776295 .

E-mail: moneimkheir62@hotmail.com

Key words: neonatal sepsis, C-reactive protein, blood culture, Sudan.

Conflict of interests: the authors declare no potential conflict of interests.

Contributions: the authors contributed equally.

Acknowledgements: the Authors express their sincere appreciation to the administration of Soba University Hospital for giving their approval and assistance in conducting the research. In addition, the Authors are grateful to the staff of the Microbiology Department and Neonatal Unit of the same Hospital for their great help.

Received for publication: 18 February 2013.

Revision received: 4 May 2013

Accepted for publication: 7 May 2013.

This work is licensed under a Creative Commons Attribution 3.0 License (by-nc 3.0).

(C) Copyright A.E. Kheir et al., 2013

Licensee PAGEPress, Italy

Healthcare in Low-resource Settings 2013; 1:e21 doi:10.4081/hls.2013.e21

finding study, conducted in the neonatal intensive care unit (NICU) at Soba University Hospital in Khartoum, Sudan, February to August 2011. All newborn babies (0-28 days) admitted to NICU at Soba University Hospital during the study period with clinical suspicion of sepsis or having risk factors for sepsis were included in the study. Specific risk factors that were used in the study are duration of membrane rupture $>18 \mathrm{~h}$, mode and place of delivery, whether labor was prolonged or precipitate, intrapartum fever, antibiotic usage during labor, history of vaginal discharge, previous sibling with neonatal sepsis in addition to the gestational age, and weight of the baby.

Pediatricians usually look for specific symptoms and signs of sepsis, i.e. symptoms like refusal of feeds, lethargy, irritability, poor cry, vomiting, diarrhea and fever. Specific signs like cyanosis, tachpnoea, apnoea, seizures, temperature instability, abdominal distension and purpura are also taken into account. Neonates with major congenital malformation or with birth weight less than $1000 \mathrm{~g}$ were excluded. A total of 70 babies were included in the study. Data were collected using a precoded and pretested specifically designed questionnaire (Appendix). At the time of admission, a complete septic screen was done, which included complete blood count, CRP, blood cul- 
ture, chest X-ray, cerebrospinal fluid (if applicable). C-reactive protein values were estimated qualitatively by the latex agglutination method, with a detection limit of $6 \mathrm{mg} / \mathrm{L}$.

The average cost of complete blood count is 60 Sudanese pound (10 US $\$$ ), blood culture is 60 Sudanese pounds (10 US \$) and qualitative CRP is 15 Sudanese pounds (2.5 US \$). Newborn babies were classified as having sepsis if they had signs suggestive of sepsis and a positive blood culture. Probable sepsis was diagnosed if they had a negative blood culture with signs suggestive of sepsis and no sepsis if there were no clinical features of sepsis with a negative blood culture. Positive blood culture was considered the gold standard against which CRP result was compared.

The following values were considered indicative of sepsis: white blood count (WBC) $\leq 5000$ or $\geq 25,000 \mathrm{~mm}^{3}$; absolute neutrophil count (ANC) $\leq 1800$ or $\geq 5000$; and CRP $6 \mathrm{mg} / \mathrm{L}$ or more.

The sensitivity, specificity, positive (PPV) and negative predictive values (NPV) were calculated by the following equations:

Sensitivity=number of true positive/number of true positive+number of false negative

Specificity=number of true negative/ number of true negative+number of false positive

PPV=number of true positive/numberof true positive+number of false positive

$\mathrm{NPV}=$ number of true negative/number of true negative+number of false negative

Data were analyzed using Statistical Package for Social Sciences (SPSS) version 17; Chi square test was used to determine the association between CRP and risk factors for sepsis and also the association between CRP and blood culture. $P$ value was set on an alpha level at 0.05 and $95 \%$ confidence limit.

Ethical clearance and approval for conducting this study was obtained from the ethical committee of Soba University Hospital. Prior informed consent was obtained from the parents of the babies participating in this study after full explanation of the study.

\section{Results}

A total of 70 babies with sepsis were admitted, males were $43(62.1 \%)$, females were 27 (37.9\%). Twenty-nine babies (41.4\%) were proven sepsis (positive blood culture plus signs of sepsis) and 41 babies (58.6\%) were probable sepsis (negative blood culture plus signs of sepsis). C-reactive protein was posi- tive in 17 babies with proven sepsis and in only 6 babies with probable sepsis. There was a significant association between CRP result and blood culture $(\mathrm{P}=0.00)$.

In our study Klebseilla pneumonae was the commonest bacteria isolated followed by Staphylococcus aureus and Escherichia coli. Figure 1 shows the percentage of babies infected with various organisms.

Lethargy and fever were the common presenting symptoms (22.9 and $15.7 \%$, respectively) followed by refusal of feed ( $8.6 \%)$, vomiting (5.7\%) and poor cry (4.3\%).

We found that Jaundice, abdominal distension, tachypnea and temperature instability were the common clinical signs (27.1, 25.7, $22.9,22.9 \%$, respectively) followed by seizures (8.6\%), purpura (5.7\%), skin mottling (1.4\%), and cyanosis (1.4\%). Of the symptomatic babies, $42.9 \%$ were CRP positive, whereas only $17.9 \%$ of the asymptomatic babies were CRP positive. There was a significant association between symptoms of sepsis and CRP results $(\mathrm{P}=0.029)$.

Higher proportion of babies with late onset sepsis were CRP positive (45.5\%), compared with those with early onset sepsis (21.6\%), and there was a significant association between onset of sepsis and CRP results $(\mathrm{P}<0.034)$.

In most of the neonates with positive CRP, their mother had regular antenatal care. There was no significant association between CRP level and antenatal care follow up $(\mathrm{P}=0.216)$. Table 1 shows the correlation between CRP and antenatal care follow up.

Out of the 70 babies, 44 were delivered by caesarian section; of these, 15 (34.1\%) were CRP positive. Our study showed that there was no significant association between CRP level and mode of delivery $(\mathrm{P}=0.775)$.

Table 2 shows the correlation between CRP and duration of membrane rupture: there was no significant association between CRP and duration of membrane rupture $(\mathrm{P}=0.137)$. There was prolonged labor in 12 (17.14\%) out of the 70 babies, whereas labor was precipitate in 3 (4.28\%). There was no significant association between CRP and history of labor ( $\mathrm{P}=0.438)$.

Interestingly enough, when taking intra-

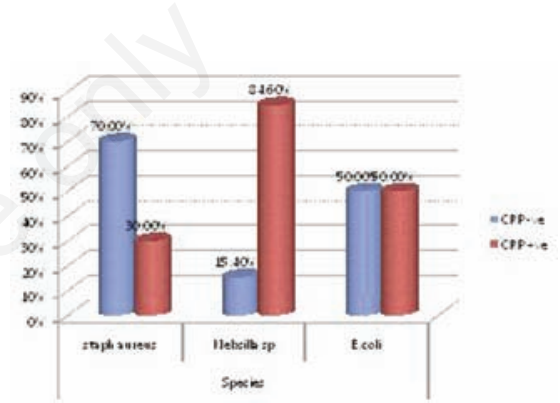

Figure 1. Babies infected with various organisms (values expressed as percentage; $\mathbf{P}=0.028)$.

Table 1. Correlation between $C$-reactive protein and regular antenatal care $(P=0.216)$.

\begin{tabular}{lcccc}
\multicolumn{2}{l}{ Regular antenatal care } & & CRP & Total \\
\cline { 3 - 4 } Yes & $\mathrm{n}$ & 44 & Positive & \\
& $\%$ & 65.70 & 23 & 67 \\
No & $\mathrm{n}$ & 3 & 34.30 & 100.00 \\
& $\%$ & 100.00 & 0 & 3 \\
\multirow{2}{*}{ Total } & $\mathrm{n}$ & 47 & 0.00 & 100.00 \\
& $\%$ & 67.10 & 23 & 70 \\
& & & 32.90 & 100.00 \\
\hline
\end{tabular}

CRP, C-reactive protein.

Table 2. Correlation between $\mathrm{C}$-reactive protein and duration of membrane rupture ( $P=$ 0.137).

\begin{tabular}{lcccc} 
Duration of membrane rupture & & CRP & & Total \\
& & Negative & Positive & \\
\multirow{2}{*}{ Normal } & $\mathrm{n}$ & 33 & 21 & 54 \\
& $\%$ & 61.10 & 38.90 & 100.00 \\
$<18 \mathrm{~h}$ & $\mathrm{n}$ & 1 & 0 & 1 \\
& $\%$ & 100.00 & 0.00 & 100.00 \\
\hline \multirow{2}{*}{$>18 \mathrm{~h}$} & $\mathrm{n}$ & 13 & 2 & 15 \\
& $\%$ & 86.70 & 13.30 & 100.00 \\
\multirow{2}{*}{ Total } & $\mathrm{n}$ & 47 & 23 & 70 \\
& $\%$ & 67.10 & 32.90 & 100.00 \\
\hline
\end{tabular}

CRP, C-reactive protein. 
partum fever as a risk factor for sepsis, our study showed that there was no significant association between CRP and intrapartum fever $(\mathrm{P}=0.615)$, again CRP level was not affected by intrapartum antibiotic usage $(\mathrm{P}=0.19)$.

Regarding the gestational age, our study showed that $31.1 \%$ of the preterm babies were CRP positive, whereas $37.5 \%$ of the term babies were CRP positive. There was no significant association between CRP and gestational age $(\mathrm{P}=0.675)$.

The correlation between CRP and birth weight was not significant $(\mathrm{P}=0.236)$. Most of the babies with sepsis had their weight ranging between 1000 and $1500 \mathrm{~g}$.

Table 3 shows the correlation between CRP and total white blood cell (TWBC) count. There was no significant correlation between CRP and TWBC $(\mathrm{P}=0.074)$. Again, there was no significant correlation between CRP and ANC $(\mathrm{P}=0.557)$.

The diagnostic value of sepsis screen parameter showed that CRP had good specificity and negative predictive value (NPV). Table 4 shows the comparative analysis of the tests used in this study.

\section{Discussion}

Neonatal sepsis with its high mortality rate still remains a diagnostic and treatment challenge for the neonatal health care providers. An early diagnosis of neonatal septicemia helps the clinician in instituting antibiotic therapy at the earliest, thereby reducing the mortality rates in the neonates. There is no single reliable test for the early definite diagnosis of neonatal sepsis, and therefore, there is a continuing search for a new infection marker. The $\mathrm{C}$ reactive protein has been the most analyzed parameter for the detection of bacterial infections for years. ${ }^{9,10}$

Severe neonatal infections are one of the most significant causes of pediatric mortality, resulting in more than 500,000 deaths each year. ${ }^{11}$ Ninety-nine percent of these deaths occur in low resource settings. ${ }^{12}$ Identifying neonates with severe infections is difficult in high resource settings, and limited laboratory capability in low resource settings makes diagnosis even more challenging. Clinical criteria for the diagnosis of neonatal sepsis have been developed and are included in the WHO Integrated Management of Childhood Illness (IMCI) program. ${ }^{13}$

In the present study an attempt has been made to evaluate the usefulness of qualitative CRP as a predictor of sepsis, and also to study the correlation between CRP, blood culture and risk factors for sepsis. This is because it is simple and cost effective.

In our study, 29 babies (41.4\%) were proven to have bacterial sepsis based on positive blood culture results. This is different from a study done in Egypt where it was found that $70 \%$ of the neonates had positive blood cultures. In the same study, the identified bacteria included Gram positive cocci, Staphylococcus epidermidis, Staphylococcus aureus, and Streptococci agalacti as the commonest organisms and this is in contrast to our study where Klebseilla pneumonae was the commonest bacteria isolated followed by Staphylococcus aureus and Escherichia coli. ${ }^{14}$

In a study conducted in Poland where 48 babies with a clinical diagnosis of sepsis were recruited, it was found that 18 (58\%) out of 31 babies with positive blood culture had increased CRP, which is similar to what obtained in our study where 17 (58\%) out of 29 babies with proven sepsis had positive CRP. ${ }^{15}$

In a study done by Dollner et al. in Norway, 6 inflammatory mediators including CRP were compared as early diagnostic tests for neonatal sepsis and the possible benefit of combining parameters was studied. C-reactive protein performed best as a diagnostic test for neonatal sepsis. Diagnostic accuracy was further improved by combining CRP and interleukin-6, whereas the other parameters added no further diagnostic information. ${ }^{16}$

In this study, CRP had sensitivity, specificity, PPV, NPV of $63,85.36,73.9,73.9 \%$, respectively, at a cut-off value of $6 \mathrm{mg} / \mathrm{L}$. These results are comparable to those reported by Abdollahi et al., i.e. lower sensitivity and higher specificity of CRP in detecting sepsis among their study group..$^{17}$ Nuntnarumit et al. in Bangkok (Thailand), reported the highest sensitivity, specificity, PPVs and NPVs. This is probably due to the quantitative sampling method which they used as compared to the qualitative method used in the present study. ${ }^{18}$

When we studied the hematological parameters for sepsis, we found that the WBC had high specificity and NPV, while the ANC showed unsatisfactory results. However, the specificities were better if combined together and can have a good NPV. This is in agreement with a study done by Varsha et al., who found that the use of multiple hematological parameters is a good diagnostic aid for both early and late neonatal sepsis. ${ }^{19}$

\section{Conclusions}

Neonatal sepsis remains a major cause of neonatal mortality and morbidity and early diagnosis and prompt treatment determine good outcome. Qualitative CRP has strong correlation with blood culture and can reliably be used as an indicator of sepsis. High cost of other inflammatory markers preclude their clinical and routine application in low resource settings. Therefore, CRP being easily measurable and more affordable, can be conveniently used as a good marker for the diagnosis of neonatal sepsis especially in developing communities with limited resources.

\section{References}

1. Aggarwal R, Sarkar N, Deorari A, Paul V. Sepsis in the newborn. Indian $\mathbf{J}$ Pediatr 2001;68:1143-7.

\begin{tabular}{lcccc} 
Test(\%) & Sensitivity & Specificity & PPV & NPV \\
CRP & 63 & 85.36 & 73.9 & 74.4 \\
TWBC & 27.60 & 87.80 & 80.77 & 81.82 \\
\hline ANC & 62.69 & 44.00 & 44.00 & 62.69 \\
\hline
\end{tabular}

PPV, positive predictive value; NPV, negative predictive value; CRP, C-reactive protein; TWBC, total white blood cell; ANC, absolute neutrophil count. 
2. Ng PC. Diagnostic markers of infection in neonates. Arch Dis Child-Fetal 2004;89: F229-35.

3. Lam HS, Ng PC. Biochemical markers of neonatal sepsis. Pathology 2008;40:141-8.

4. Bhatti M, Chu A, Hageman J, et al. Future directions in the evaluation and management of neonatal sepsis. Neoreviews 2012; 13:e103.

5. Hawk M. C-reactive protein in neonatal sepsis. Neonatal Netw 2008;27:117-20.

6. Philip AG, Mills PC. Use of C-reactive protein in minimizing antibiotic exposure. Pediatrics 2000;106:e4.

7. Chacko B, Sohi I. Early onset neonatal sepsis. Indian J Pediatr 2005;72:23-6.

8. Mehr S, Doyle LW. Cytokines as markers of bacterial sepsis in newborn infants: a review. Pediatr Infect Dis J 2000;19:87987.

9. Manneret G, Labaune JM, Isaac C, et al. Procalcitonin and C-reactive protein levels in neonatal infections. Acta Paediatr 1997;86:209-12.
10. Chiesa C, Signore F, Assumma M, et al. Serial measurements of the C-reactive protein and interleukin 6 in the immediate postnatal period: the reference intervals and the analysis of the maternal and the perinatal confounders. Clin Chem 2001;47:1016-22.

11. Black RE, Cousens S, Johnson HL, et al. Global, regional, and national causes of child mortality in 2008: a systematic analysis. Lancet 2010;375:1969-87.

12. Thaver D, Zaidi AK. Burden of neonatal infections in developing countries: a review of evidence from community-based studies. Pediatr Infect Dis J 2009;28:3-9.

13. Gove $S$. Integrated management of childhood illness by outpatient health workers: technical basis and overview. The WHO working group on guidelines for integrated management of the sick child. Bull World Health Organ 1997;75(Suppl.1):724.

14. Boraey N, Sheneef A, Mohamed MA, Yousef LM. Procalcitonin and C-reactive protein as diagnostic markers of neonatal sepsis. Aust J Basic Appl Sci 2012;6:108-14.

15. Kawczynski P, Piotrowski A. Procalcitonin and C-reactive protein as markers of neonatal sepsis. Ginekol Pol 2004;75:43944.

16. Dollner H, Austgulen R, Vatten L. Early diagnostic markers for neonatal sepsis. J Clin Epidemiol 2001;54:1251-7.

17. Abdollahi A, Shoar S, Nayyeri F, Shariat M. Diagnostic value of simultaneous measurement of Procalcitonin, Interleukin-6 and hs-CRP in prediction of early-onset neonatal sepsis. Mediterr J Hematol Infect Dis 2012;4:e2012028.

18. Nuntnarumit P, Pinkaew 0, Kitiwanwanich S. Predictive values of serial Creactive protein in neonatal sepsis. J Med Assoc Thai 2002;85:1151-8.

19. Varsha, Rusia U, Sikka M, et al. Validity of hematologic parameters in identification of early and late onset neonatal infection. Indian J Pathol Micr 2003;46:565-8. 